\title{
Employability und Fähigkeiten für die Zukunft der Arbeit am Beispiel des Maschinenbauwesens
}

\author{
Marija Stambolieva
}

\section{Zusammenfassung}

Dieser Artikel untersucht die Beziehung zwischen Arbeitsmarkt und Hochschulbildung sowie den Einfluss von Technologie auf Arbeitsplätze, Berufe und Qualifikationen, insbesondere aus der Perspektive der Humankapitaltheorien. Die Ergebnisse unserer qualitativen Untersuchung unter Arbeitgebenden aus dem Maschinen- und Anlagenbau und der Automobilindustrie zeigen, dass es eine klare Erwartungshaltung an den Wandel der Berufsprofile der Ingenieurwissenschaftler*innen und die damit verbundenen Fähigkeitsanforderungen aufgrund der Digitalisierung gibt. Damit gewinnt die Dimension der Beschäftigungsbefähigung (Employability) bei der Curriculumentwicklung an Bedeutung. Einschränkend wirken dabei die ressourcenintensive Einbeziehung der Sichtweisen der Arbeitgebenden und die uneinheitliche Operationalisierung der Qualifikations- und Kompetenzanforderungen, sowohl in unterschiedlichen Studien als auch in den Curricula. Die Orientierung am aktuellen HQR-Kompetenzmodell wäre für die Vergleichbarkeit der Arbeitsmarktchecks sinnvoll.

\section{Schlüsselwörter}

Employability • Digitalisierung • Maschinenbauingenieur*in • Arbeitgebende • Kompetenzorientierung • Curriculumentwicklung • Hochschulqualifikationsrahmen (HQR)

M. Stambolieva ( $\square)$

Hochschule Osnabrück, Osnabrück, Deutschland

E-Mail: m.stambolieva@hs-osnabrueck.de 


\section{$1 \quad$ Einleitung}

Zwei Arten von Diskursen dominieren im Bereich der Hochschulbildung und des Arbeitsmarktes: der Diskurs zur Curriculumentwicklung und der Diskurs zur Employability. Sie finden oft parallel zueinander statt: Der eine Diskurs thematisiert die Kompetenzprofile im Hochschulkontext, der andere die berufliche Perspektive auf die Kompetenzbildung. Eine wichtige neue Dimension in der Diskussion bringen der technologische Wandel durch die Digitalisierung und seine Auswirkungen mit. Aufgrund der hohen Geschwindigkeit ihres Einflusses könnte sie sich als derjenige Faktor erweisen, der zu einer stärkeren Verknüpfung zwischen den Hochschul- und Arbeitsmarktdiskursen beiträgt. Deshalb wird im Artikel argumentiert, dass die Beschäftigungsbefähigung als ein ebenso wichtiger Aspekt im Studium betrachtet werden sollte.

Der Artikel ist wie folgt strukturiert: Abschnitt eins gibt einen Überblick über die einschlägige Literatur und stellt die Forschungsfragen vor. Abschnitt zwei fasst das Forschungsdesign und die Methoden zusammen. Abschnitt drei stellt die Ergebnisse unserer qualitativen Untersuchung vor und diskutiert die Implikationen. Abschnitt vier fasst die Ergebnisse zusammen und schlägt zukünftige Forschungsschritte vor.

\section{$2 \quad$ Was sagt die Literatur?}

\subsection{Digitalisierung}

Die Literatur zur technologischen Transformation thematisiert größtenteils die Arbeitsplatzeffekte auf der Grundlage der Qualifikationsstruktur der Beschäftigten. Obwohl sich die Studien in den Klassifizierungsstandards unterscheiden, haben sie alle gemeinsam, dass sie einen Rückgang der routinemäßigen Berufe zeigen, die mit einem mittleren Qualifikationsniveau assoziiert sind, und einen Anstieg sowohl der nichtroutinemäßigen manuellen als auch der nichtroutinemäßigen kognitiven Berufe, die mit einem niedrig beziehungsweise hoch qualifizierten Niveau assoziiert sind (Goos et al. 2014; Kurer und Palier 2019; OECD 2019). In ähnlicher Weise macht ein anderer Teil der Literatur Vorhersagen über Arbeitsplätze, die von der Automatisierung bedroht sind, indem er Berufe analysiert und den Tätigkeitsinhalt der Arbeitsplätze inspiziert (Acemoglu und Autor 2011; Nedelkoska und Quintini 2018). Während die Schätzungen erheblich variieren, haben die Studien die Ansicht gemeinsam, dass komplexere Arbeitsaufgaben ein höheres Qualifikationsniveau erfordern, das in der Regel 
durch eine Hochschulausbildung erreicht wird. Von daher sind höher qualifizierte Arbeiter*innen schwieriger zu ersetzen. Dies führt uns zu den Fragen: Wie sicher sind hoch qualifizierte Arbeitsplätze? Welche Art von Fertigkeiten und Fähigkeiten sind notwendig, um den Anforderungen zukünftiger Arbeitsplätze gerecht zu werden? Und welche Rolle müssen die Hochschulen übernehmen?

\title{
2.2 Employability und die Hochschulperspektive
}

Seit der Einleitung des Bologna-Prozesses auf europäischer Ebene ist Employability ein Schlüsselbegriff geworden. Zugleich bleibt er ein umstrittenes Konzept. Insbesondere die deutsche Übersetzung registriert verschiedene Begriffe (vgl. Schubarth et al. 2014), bis sich die Hochschulrektorenkonferenz (HRK) für die Verwendung des Begriffs Beschäftigungsbefähigung mit folgender Bedeutung ausgesprochen hat:

\begin{abstract}
„Beschäftigungsbefähigung von Hochschulabsolventen bedeutet, dass diese auf Basis wissenschaftlicher Bildung (fachliche und überfachliche Kompetenzen sowie berufsfeldbezogene Qualifikationen) eine qualifizierte Beschäftigung aufnehmen, diese halten und sich neue Beschäftigungsfelder erschließen können“ (HRK 2020a).
\end{abstract}

Diese Definition stellt auch einen Versuch dar, die verschiedenen Debatten um die Ziele der Hochschulbildung zwischen Vermittlung wissenschaftlichen Wissens, Kompetenzorientierung und beruflicher Qualifizierung in Einklang zu bringen.

Traditionell vermitteln Hochschulen fachspezifisches Wissen und haben wenig Erfahrung in der Förderung von Kompetenzen (wie methodische, soziale, interkulturelle Kompetenzen) (EHEA 2016). Andererseits sind die europaweit initiierten Bologna-Reformen kompetenzorientiert und erfordern, „Studierende in die Lage zu versetzen, [...] nicht nur fachwissenschaftliche Fähigkeiten, sondern auch Einstellungen und Werte sowie überfachliche Schlüsselkompetenzen möglichst integrativ zu entwickeln“ (HRK 2020b). Trotz der Uneinheitlichkeit des Kompetenzbegriffes (vgl. Erpenbeck et al. 2017; Lautenbach et al. 2017) und insbesondere aufgrund der Uneinigkeit, was als „Schlüsselkompetenz“ bezeichnet werden kann (Schubarth et al. 2014, S. 52), orientieren sich die Hochschulen an den Vorgaben der Kultusministerkonferenz (KMK). So klassifiziert der 2017 von der KMK beschlossene Qualifikationsrahmen für Deutsche Hochschulabschlüsse (HQR) folgende Kompetenzdimensionen: Fachkompetenz (Wissen und Verstehen), Methodenkompetenz (Einsatz, Anwendung und Erzeugung von Wissen), Selbstkompetenz (wissenschaftliches Selbstverständnis, Professionalität) und 
Sozialkompetenz (Kommunikation und Kooperation) (KMK 2017a, b, S. 4 und 9). Die konkrete Kompetenzprofilerstellung eines Studiengangs ist den Hochschulen überlassen. Die Entwicklung von Kompetenzmodellen innerhalb der Curricula ist problembehaftet, verbunden mit der unterschiedlichen Konzeptionierung und empirischen Erfassung von Kompetenzen oder der unterschiedlichen Handlungsorientierung in der Curriculumentwicklung (vgl. Dilger et al. 2012; Gerholz und Sloane 2011; Gössling und Luft 2019).

Weitere Unklarheiten entstehen, wenn wir nicht nur über Kompetenzprofile sprechen, sondern auch die konkreten Berufsprofile und berufsfeldbezogenen Qualifikationen berücksichtigen, für die die Studiengänge qualifizieren müssen. So ist der Berufsbezug unterschiedlicher Studiengänge sehr unterschiedlich und reicht von einem festen Berufsbild, wie zum Beispiel Medizin oder Lehramt über eine Bandbreite beschreibbarer Berufe und Tätigkeitsfelder, wie beispielsweise Rechtswissenschaften, Betriebswissenschaften und Ingenieurwissenschaften, bis zu keinen konkreten Berufsbildern und sehr vielfältigen Tätigkeitsfeldern in den Geistes- und Sozialwissenschaften (Griepentrog 2010 in Hessler 2013; Eimer et al. 2019). Veränderte Aufgabeninhalte einzelner Arbeitsplätze im Zusammenhang mit dem technologischen Wandel stellen eine zusätzliche Herausforderung dar.

Während die Bologna-Erklärung darauf abzielte, Employability zu fördern, macht das Ministerkommuniqué von Jerewan 2015 mit Hinsicht auf ,rapidly changing labour markets - characterized by technological developments, the emergence of new job profiles, and increasing opportunities for employment and self-employment“" (EHEA 2015) Employability zu einem der Hauptziele des Europäischen Hochschulraums. Allerdings erweist sich die konkrete Operationalisierung von Employability in den Curricula, bezüglich der Identifikation von potenziellen Berufsfeldern, der Differenzen nach Hochschulart und nach Konkretheit des Berufsfeldes sowie wegen mangelnder Konzeptionierung und empirischer Erfassung von Employability und Kompetenzentwicklung als schwierig (vgl. Schubarth et al. 2014; European Commission/EACEA/Eurydice 2018; Eimer et al. 2019). Angesichts der verstärkten politischen Verpflichtungen sowie des Arbeitsmarktwandels stellt sich die legitime Frage, wie die Arbeitgebendenperspektive in zukünftigen Curriculumentwicklungen berücksichtigt werden kann.

\subsection{Arbeitgebendenperspektive}

Verschiedene Studien versuchen zu prognostizieren, welche Fähigkeiten in Zukunft aus Sicht der Arbeitgebenden wichtig werden (WEF 2016; acatech 2016; 
Stifterverband für die Deutsche Wissenschaft 2016). Weitere Studien betonen besonders die Bedeutung der Datenkompetenz (Schüller und Busch 2019; Schüller et al. 2019). Die Studien unterscheiden sich nach Methodik und Befund oder Klassifizierung der Kompetenzen. Laut dem Stifterverband für die Deutsche Wissenschaft (2016) besteht das Kompetenzprofil für die Arbeitswelt 4.0 aus Fachkompetenzen, berufsorientierten Kompetenzen und persönlichkeitsbildenden Kompetenzen. Die Studie kommt zum Ergebnis, dass überfachliche Kompetenzen und spezialisiertes Fachwissen an Bedeutung gewinnen, während Grundlagenund Methodenkompetenzen einen geringeren Zuspruch finden. Diese Befragungsergebnisse stehen teilweise in Widerspruch zu anderen, fachspezifischen Studien, die zum Beispiel das Grundlagenfach- und -methodenwissen nach wie vor für wichtig halten (vgl. Heidling et al. 2019).

Im Bereich der Ingenieurwissenschaften gibt es eine begrenzte Anzahl von Studien, die sich mit konkreten Qualifikations- und Berufsprofilen befassen (Dengler und Matthes 2018; Pfeiffer und Suphan 2018; Gottburgsen et al. 2019; Heidling et al. 2019). Einige Studien erkunden das Substituierbarkeitspotenzial bestimmter Tätigkeitsfelder in unterschiedlichen Berufen sowie die Entstehung neuer Berufe aufgrund der Digitalisierung (Dengler und Matthes 2018). Andere weisen auf die Einschränkungen des Routineansatzes hin (Pfeiffer und Suphan 2018).

Aufgrund von Studierendenbefragungen sowie Befragungen der Berufseinsteiger*innen des Vereins Deutscher Ingenieure (VDI) ermitteln Gottburgsen et al. (2019) die Kompetenzen der Ingenieur*innen für eine digitalisierte Arbeitswelt. Sie heben insbesondere sogenannte digitale Fachinhalte hervor, die als

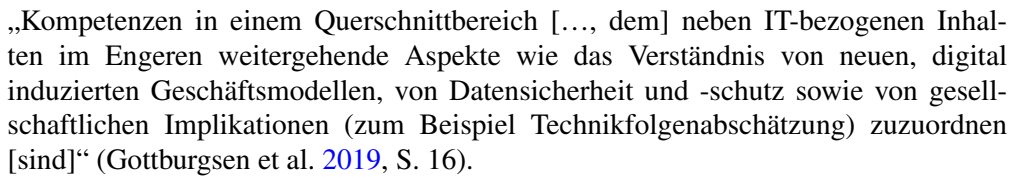

Heidling et al. (2019) versuchen, ein vollständiges Sollprofil der Ingenieur*innen im Maschinen- und Anlagenbau zu erstellen. Es soll aus einem grundlegenden fachlich-methodischen Kern und inhaltlich flexiblen fachlichen und überfachlichen Ergänzungen bestehen. Zum grundlegenden fachlich-methodischen Kern zählen laut der Studie Grundkenntnisse in einer Fachdisziplin (zum Beispiel Maschinenbau), erweitert durch Grundlagenkenntnisse in Informatik und Data Science sowie Methodenkompetenz des Prozess- und Systemdenkens. Dies ist gleichzeitig die einzige fachspezifische Studie, die sich an die Kompetenzdimensionen des HQRs orientiert. 
Dieser kurze Literaturüberblick zeigt, dass kein einheitliches Bild der Qualifikations- und Kompetenzanforderungen besteht. Die Quellen erwecken oft den Eindruck, eine willkürlich erstellte Wunschliste zu präsentieren. Zudem fehlen Definitionen. Die Anforderungen werden oft nur genannt, ohne Hintergrundinformationen oder Operationalisierung und das Verständnis seitens der befragten Personen. Fachspezifische Studien sind selten.

\section{$3 \quad$ Hintergrund, theoretischer Rahmen und Methodologie}

\subsection{Hintergrund}

Es ist notwendig, den beruflichen Wandel durch die Digitalisierung systematisch zu untersuchen. Unsere Studie ${ }^{1}$ untersucht den Ingenieursberuf, insbesondere am Beispiel des Maschinenbaus, und seine Berufs- und Tätigkeitsfelder mit der Absicht, den Ist-Zustand systematisch zu erfassen und die Entwicklungen in diesem Berufsfeld festzuhalten. Dies tun wir aus zwei Gründen: zum einen aus dem Interesse der Fakultätsleitung, die Zukunftsfähigkeit unserer Studiengänge zu prüfen, und zum anderen, weil der Maschinen- und Anlagenbau sowie verwandte Branchen, wie zum Beispiel die Automobilindustrie, die größten industriellen Arbeitgebenden in Deutschland ${ }^{2}$ sind und die dort gesammelten Erkenntnisse einen relevanten Indikator für den Ingenieursberuf darstellen.

Der Artikel trägt auf zwei Arten zur Literatur bei: Erstens präsentiert er Belege für sich ändernde Kompetenzanforderungen aus einer berufsspezifischen Perspektive. Die meisten Studien machen allgemeine Vorhersagen und sind zudem methodisch sehr unterschiedlich und daher schwer zu vergleichen. Die einzige Studie, die ein Berufsfeld (Maschinenbau) systematisch in den Blick nimmt, ist die von Heidling et al. (2019). Wir vergleichen daher die Ergebnisse beider Studien. Zweitens ist unsere Studie die erste, die explizit Bezug auf den HQR als wichtigen Indikator für die Kompetenzermittlung nimmt.

\footnotetext{
${ }^{1}$ Die Untersuchung erfolgte im Rahmen des Projektes „Qualität Plus der Fakultät der Ingenieurswissenschaften und Informatik“ an der Hochschule Osnabrück.

${ }^{2} \mathrm{Im}$ Jahr 2018 waren rund 1.065.000 Beschäftigte im deutschen Maschinenbau tätig (Statista 2020).
} 


\subsection{Theoretischer Rahmen}

Die Theorie des Humankapitals stellt Bildung in den Mittelpunkt der wirtschaftlichen Entwicklung. Sie wurde ursprünglich Becker (1962) zugeschrieben und legt hauptsächlich nahe, dass Bildungsinvestitionen in das eigene Humankapital, wie Wissen und Fähigkeiten, den wirtschaftlichen Ertrag fördern. Kritiker*innen finden, dass sie Individuen auf ihren wirtschaftlichen Wert reduziert (Gillies 2011) oder andere Faktoren, die die Position des Einzelnen auf dem Arbeitsmarkt bestimmen können, wie beispielsweise strukturelle Diskriminierung (vgl. Arrow 1973), die Bedeutung des Sozialkapitals (vgl. Bourdieu 1983), die durch die institutionellen Arrangements des Arbeitsmarktes und des Bildungssystems auferlegten Beschränkungen (vgl. Allmendinger 1989) oder persönliche Anpassungsund Identitätsfragen (vgl. Fugate et al. 2004), vernachlässigt.

Wir beziehen uns auf die Humankapitaltheorie, da sie Bildung als einen wichtigen Prädiktor für die Verbesserung der Arbeitsmarktposition einer Person betrachtet. Insbesondere die Variante des Signalansatzes (Spence 1973) unterstreicht die formalen Bildungsnachweise als eine wichtige Informationsquelle für Arbeitgebende bezüglich der individuellen Produktivität. Speziell in Deutschland werden formale Qualifikationen als Hauptkriterium für das Kompetenzniveau einer Person gesehen (Allmendinger und Leibfried 2003). Daher argumentieren wir, dass die Rolle des formalen Kompetenzerwerbs besonders im Zuge der dynamischen Veränderungen, die durch die Expansion der „Wissensökonomie“ oder die Digitalisierung hervorgerufen werden, überprüft werden muss. Abb. 1 zeigt unser Modell, das die Verbindung zwischen Curriculumentwicklungen und Änderungen auf dem Arbeitsmarkt untersuchen möchte.

Einerseits gestalten die Hochschulen die Curricula ihrer Studiengänge, indem sie die berufsfeldbezogenen Qualifikationsziele in Form von wissenschaftlichen Grundlagen, fachlichen, methodologischen, sozialen und Selbstkompetenzen, welche Studierende im Laufe des Studiums erwerben sollen, und die zu erwartenden Lernergebnisse, das heißt Kenntnisse, Fertigkeiten und Fähigkeiten, die die Studierenden in einem bestimmten Modul oder einer Lehrveranstaltung erwerben müssen, definieren (KMK 2017a, b). Die 2017 überarbeitete Version des HQR aus dem Jahr 2005, einschließlich Begrifflichkeit und Zuordnung der Kompetenzkonstrukte, stellt neben der problematischen Operationalisierung von Kompetenzmodellen in konkrete Curricula eine zusätzliche Herausforderung für den Aushandlungsprozess im Rahmen der Curriculumentwicklung dar, da alte Curricula vergleichsweise weiterentwickelt, neu interpretiert und nicht zuletzt empirisch überprüft werden müssen. 


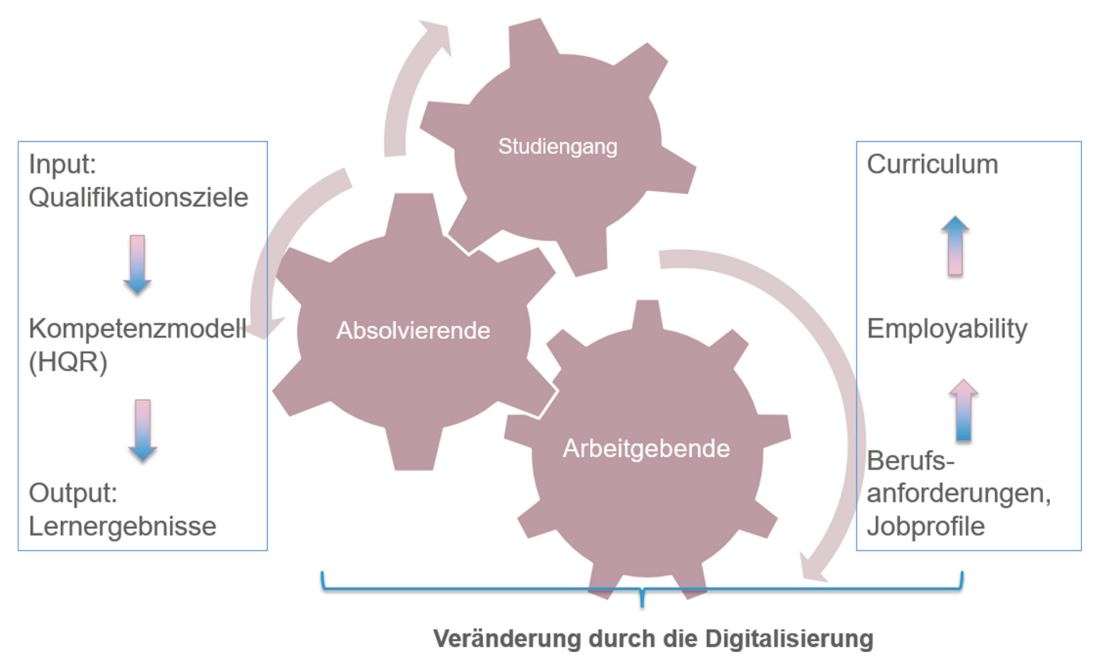

Abb. 1 Untersuchungsmodell. (Eigene Darstellung)

Andererseits evaluieren wir die aktuellen Entwicklungen auf dem Arbeitsmarkt und ihre Rolle in der Curriculumentwicklung. Die Ausweitung der Rolle der Hochschulen von wissenschaftlicher Qualifizierung bis zur Befähigung zur Berufstätigkeit und zum lebenslangen Lernen ist immer wieder diskutiert worden und während eine starke Einbindung der Arbeitgebendenperspektive nicht unkritisch ist, gibt es auch zunehmend Forderungen nach einem systematischen Überdenken der Verbindungen zwischen den Hochschulen und der Arbeitswelt (vgl. Gerholz und Sloane 2011; Schubarth et al. 2014; Teichler 2018; Eimer et al. 2019). Wir fragen daher die Arbeitgebenden nach ihren wahrgenommenen und erwarteten Anforderungen an die Maschinenbauingenieur*innen im Hinblick auf die digitale Transformation. Dabei konzentrieren wir uns auf die Humankapitaldimension der Employability. Da die Forschungsfragen entlang des HQR-Kompetenzrahmens strukturiert sind, erwarten wir relevantes Feedback für die Ingenieurstudiengänge. 


\subsection{Methodologie}

Das Untersuchungsvorhaben kombiniert Literaturrecherche, qualitative und quantitative Methoden. Die qualitative Datenerhebung erfolgte durch halbstrukturierte Interviews (Einzelinterviews und Gruppendiskussionen) mit Unternehmensvertreter*innen aus der Region (Osnabrücker-, Münster- und Emsland). Daten zur Digitalisierung und die damit verbundenen Transformationen der Produkte, Produktionsprozesse und Arbeitsorganisation in den Unternehmen, zu Veränderungen in den Tätigkeiten sowie in den Kompetenzanforderungen an die Maschinenbauingenieur*innen und zu den zukünftigen Erwartungen an die Hochschulen als Bildungsanbieter wurden erhoben. Die Stichprobenauswahl erfolgte nach einer Kombination der Methoden des selektiven- und des fallkontrastiven Samplings. Die bestehende Vielfalt nach Branchen, Unternehmensgröße, Digitalisierungsgrad sowie Tätigkeitsbereich der befragten Personen sollte abgebildet werden.

Zwischen Mai und November 2019 fanden 20 teilstrukturierte Interviews in zwölf Unternehmen statt. Kleine, mittlere und große Unternehmen aus dem Maschinen- und Anlagenbau sowie der Automobilindustrie wurden befragt. Sowohl Maschinenbau- und Elektroingenieur*innen als auch Führungskräfte waren unter den Interviewten. Die befragten Unternehmen bilden das gesamte Digitalisierungsspektrum ab und ordnen sich von Anfänger*innen bis zu Fortgeschrittenen ein. Die Interviews wurden mit einem Aufnahmegerät dokumentiert und im Anschluss mithilfe der Software MAXQDA wörtlich und anonymisiert transkribiert, codiert und ausgewertet. Geplant ist eine Ergänzung der qualitativen Untersuchung durch eine Onlinebefragung von Unternehmensvertreter*innen. Durch die größere Stichprobe wird eine weitere Überprüfung der aus der qualitativen Untersuchung abgeleiteten Annahmen angestrebt.

\section{$4 \quad$ Ergebnisse: Wahrnehmungen und Erwartungen der Arbeitgebenden}

Im folgenden Abschnitt thematisieren wir die Fragen bezüglich der aktuellen und erwarteten Tätigkeitsveränderungen bei den Maschinenbauingenieur*innen und danach gehen wir auf die Fertigkeits- und Fähigkeitsanforderungen ein. Abschließend diskutieren wir über die Auswirkungen der Erwartungen der Arbeitgebenden auf die Hochschulcurricula. 


\subsection{Veränderungen der Tätigkeitsbereiche der Maschinenbauingenieur*innen}

Die Berufsgruppe der Ingenieur*innen ist historisch betrachtet heterogen gewesen und eine präzise Erfassung der Tätigkeitsfelder ist problematisch (vgl. Kaiser 2006). Eine der größten Veränderungen im Ingenieursberuf erfolgte dank der Einführung des computergestützten Designs, das erstmals Anwendung in den Bereichen der Konstruktion und des technischen Zeichnens findet und schrittweise auf die Produktion erweitert wird (ebenda). Der Einsatz von Computern und Softwareprogrammen hat viele Tätigkeitsbereiche des Ingenieursberufs wie Konstruktion, Fertigung, Planung und Überwachung, Vertrieb und anderes durchdrungen und zusätzlich die Automatisierung in der Produktion vorangetrieben. Auch wenn Veränderungen den Ingenieursberuf prägen, ist heute die Rede von einer neuen Qualität des Wandels aufgrund von digitalen Technologien und der Umstellung auf Industrie $4.0^{3}$.

Trotz der Vielfalt bei der Umsetzung neuer Technologien in den Unternehmen sowie der Vielfältigkeit des Ingenieursberufs generell und des Maschinenbauingenieursberufs speziell werden insbesondere kollaborative Roboter, selbst lernende Computerprogramme sowie erste Anwendungen von 3D-Druck und virtueller Realität als Technologien identifiziert, die die beruflichen Tätigkeiten verändern (Dengler und Matthes 2018; Bundesagentur für Arbeit 2020). Dies weist auf die Faktoren hin, die bestimmte Arbeitsaufgaben beeinflussen oder im schlimmsten Fall ersetzen, beschreibt aber nicht den Inhalt der tatsächlichen Veränderung.

Aus diesem Grund befragten wir die Arbeitgebenden, worin die typischen Aufgaben der Maschinenbauingenieur*innen in ihrem Unternehmen bestehen und wie sich die Aufgabenprofile durch die Digitalisierung verändern. Abb. 2 systematisiert alle Antworten pro Kategorie (typische Aufgaben sowie alle wahrgenommenen Veränderungen im Tätigkeitsbereich der Maschinenbauingenieur*innen). Unabhängig vom Digitalisierungsgrad sind sie sich einig, dass sich die Aufgabenprofile der Maschinenbauingenieur*innen verändern, und zwar in Bezug auf die Einführung digitaler Technologien:

„Es gibt keinen reinen Maschinenbau oder wenig reinen Maschinenbau noch. Fast alles hat irgendwo mittlerweile einen Sensor, wird mitüberwacht, es muss mitbedacht werden.“ (Interviewpartner*in 4, 2. Quartal 2019)

\footnotetext{
${ }^{3}$ Der Begriff Industrie 4.0 bezieht sich auf, ,eine hochautomatisierte und vernetzte industrielle Produktions- und Logistikkette, [in der] virtuelle und reale Prozesse auf der Basis sogenannter cyberphysischer Systeme verschmelzen“ (BMAS 2017, S. 200).
} 


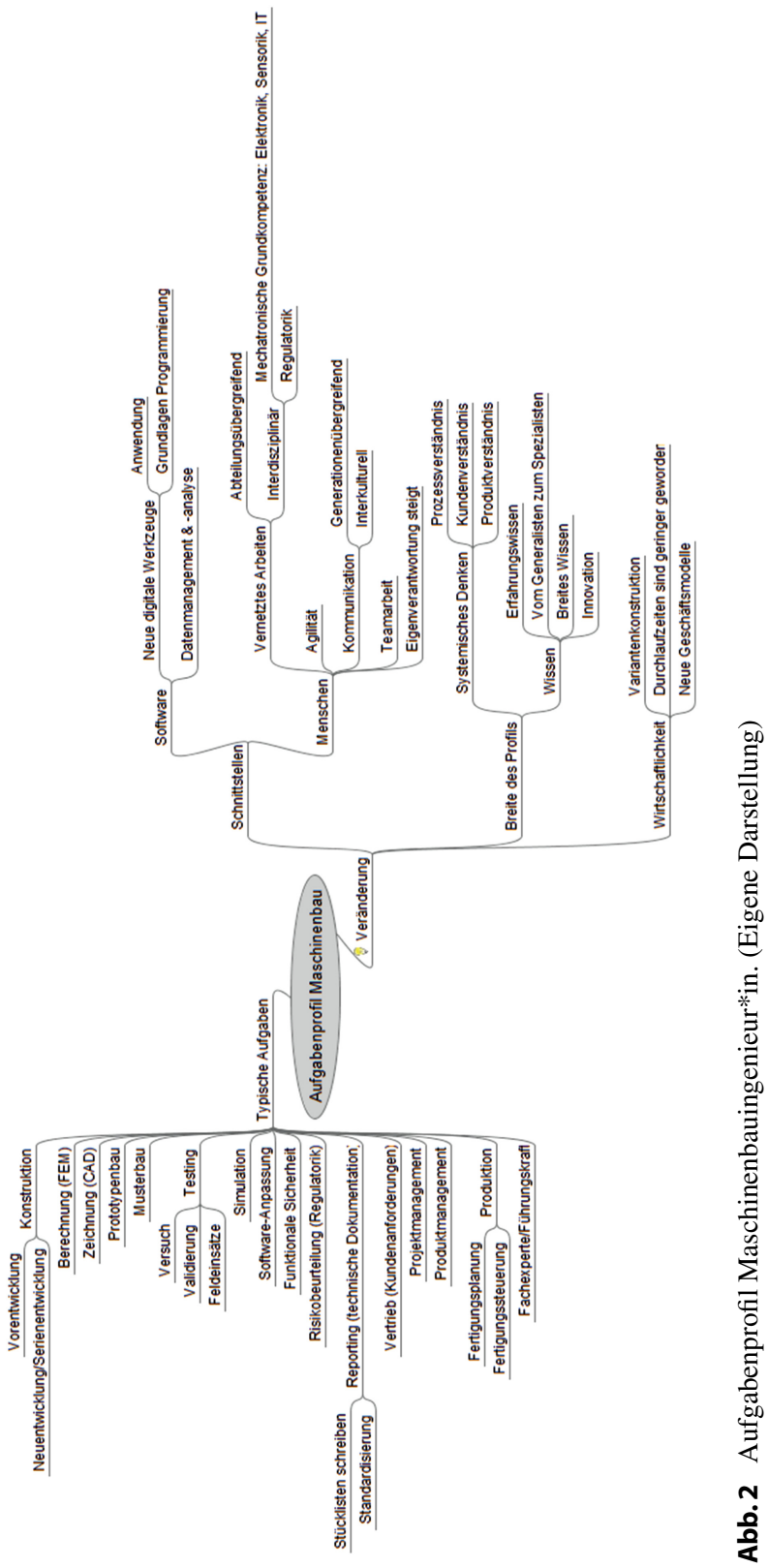


Produktionsprozesse und die gesamte Wertschöpfungskette verändern sich:

„Der Maschinenbauer ${ }^{4}$ darf nicht nur an Maschinenbau denken, sondern er muss eigentlich von der Maschine bis zum Smartphone denken." (Interviewpartner*in 1, 3. Quartal 2019)

Einerseits übernehmen die digitalen Werkzeuge einige der klassischen Aufgaben der Maschinenbauingenieur*innen, wie beispielsweise Berechnungen oder Zeichnungen, und erleichtern damit deren Arbeit. Auf der anderen Seite kommen andere Arten von Aufgaben dazu, wie beispielsweise im Bereich der Anwendung oder Grundlagenprogrammierung.

Die Rolle der Daten wurde insbesondere hervorgehoben. Neue Technologien ermöglichen die Erfassung, Speicherung, Übermittlung und Auswertung von gröBeren Datenmengen und das wirkt sich auf die Produkte, Produktionsprozesse und Geschäftsmodelle aus. Darauffolgend:

„[...] da braucht es auch Leute, die eine Datenanalyse durchführen können. [...] Wir können viel mit Künstlicher Intelligenz abdecken, irgendwelche Algorithmen dann machen, um diese Datenmengen überhaupt einigermaßen beherrschen zu können, aber ich bin nach wie vor der Meinung, dazu, genau dazu brauchen wir Ingenieure, die das nochmal hinterfragen, die sagen: ,Boah, das ist jetzt, das ist so oder ist auch nicht so. Dieses Bauchgefühl, aber auch das erfahrene Wissen, was man hat, ich glaube, das ist ganz wichtig." (Interviewpartner*in 5, 3. Quartal 2019)

Die Breite des Profils ändert sich ebenfalls. Eine systematische Herangehensweise an die Aufgaben und Teamarbeit an den Schnittstellen zu anderen Fachdisziplinen unter Berücksichtigung der betriebswirtschaftlichen Aspekte und Geschäftsmodelle wird erwartet:

„Es ist eine Art Komplexitätsfalle, in die wir da hineinlaufen, weil, also bei uns war das klassisch so, sage ich mal, vor 15, 20 Jahren, da hat ein Konstrukteur irgendwo eine komplette Maschine entwickelt mit allem Pipapo. Das war einfach hier üblich. Da gibt es einfach ein paar Namen, wo man sagt: Den verbindet man mit der Maschine, das war seine Maschine. Die hat er komplett entwickelt. Das ist heute komplett vorbei. Wir haben absolute Teamarbeit. Wir haben immer für jede Maschinentype eigentlich einen Elektroniker heute schon standardmäßig und einen Mechaniker sozusagen, die auf jeden Fall die Köpfe als Systemingenieure zusammen haben. "(Interviewpartner*in 1, 3. Quartal 2019)

\footnotetext{
${ }^{4}$ Die Befragten verwenden die männliche Form.
} 
Eine kleine Minderheit, die behauptet, dass die Tätigkeiten gleichgeblieben sind, schließt dies bei digitalen Produkten aus, die ihres Erachtens nicht zur Maschinenbaubranche gehören:

„Ich glaube, da wird sich gar nicht so wahnsinnig viel ändern in der Art und Weise, wie die, die Maschinen als Maschinen gebaut werden. Da gibt es mit Sicherheit auch digitale Dinge, was weiß ich, die Art und Weise, mit welchen, welcher Software man arbeitet, oder welche Informationen man dafür hat. [...] Ist halt eine Evolution, aber ich glaube nicht, dass da jetzt ein radikaler Umbruch stattfindet. Bei den digitalen Produkten sieht das schon ein bisschen anders aus. Ich glaube, man muss wirklich lernen, wie man digitale Produkte entwickelt. Das ist diametral anders, als wirklich Maschinen zu entwickeln. Also wirklich ganz kurze Entwicklungszyklen runterbrechen auf Funktionen, diese schnell ausprobieren, Prototypen bauen, Ideen generieren, Ideen verwerfen. Also eine sehr hohe Agilität, die da notwendig ist." (Interviewpartner*in 7, 3. Quartal 2019)

\subsection{Kompetenzanforderungen}

Anhand der HQR-Kompetenzklassifizierung fragten wir die Arbeitgebenden, welche Fach-, Methoden- sowie Sozial- und Selbstkompetenzen die Maschinenbauingenieur*innen mitbringen müssen, und baten sie, diese nach Wichtigkeit zu priorisieren.

Die Ergebnisse zeigen ein differenziertes Bild. Während einige Unternehmen eine bestimmte Kompetenz als wichtig hervorheben, behaupten andere Unternehmen das Gegenteil oder finden alle Fähigkeiten gleich relevant. Unklar ist es auch, welches Kompetenzniveau im Sinne der Bloomschen Taxonomie ${ }^{5}$ vorausgesetzt wird. Es zeigte sich, dass die unterschiedliche Wertung mit den unterschiedlichen Aufgabenbereichen, Positionen (Fach- oder Führungskraft) und Alter der Mitarbeitenden zu tun hat und sich durch den spezifischen Unternehmensfokus erklären lässt.

Alle Befragten sind sich einig, dass sowohl mathematische und physikalische Grundlagenkenntnisse als auch klassische Fachkompetenzen, die „,in einem Maschinenbaustudium einfach nicht wegzudenken sind", wie Konstruktionslehre, technische Mechanik, Werkstoffkunde, nach wie vor „das A und O“ sind und nicht durch die Digitalisierung ersetzt werden. Die Befragten gehen davon aus,

${ }^{5}$ Die Bloomsche Lernzieltaxonomie besteht aus sechs Hauptkategorien: Wissen, Verstehen, Anwendung, Analyse, Synthese und Bewertung, wobei die Kategorien nach Wissen als Fähigkeiten und Fertigkeiten dargestellt werden (Schüller et al. 2019, S. 27). 
dass eine grundlegende Fachkompetenz nach dem Studium vorhanden ist, die im Unternehmen durch weitere Spezialisierungen ausgebaut werden kann.

Die Frage nach der erwarteten Methodenkompetenz lässt sich am schwierigsten beantworten. Das hängt mit dem unterschiedlichen Verständnis, was diese Fähigkeit ausmacht, zusammen. Während einige Unternehmensvertreter*innen damit eine empirisch-wissenschaftliche Herangehensweise, analytische und Problemlösungskompetenz verbinden, haben andere die Beherrschung von Prozessoptimierungs-, Simulations-, digitalen Entwicklungs-(FEM)- oder Predictive-Maintenance-Methoden und -Tools im Blick. Einige Befragten erwähnten eine erhöhte Anwendung von agilen Arbeitsmethoden im Rahmen von Projektarbeiten.

Laut den Befragten wird Teamarbeit immer wichtiger und deshalb rücken Sozialkompetenzen in den Vordergrund. Die vorherrschende Wahrnehmung ist, dass das

„Ingenieurbild vor 30 Jahren noch umgekehrt war. Da stand immer das Fach ganz oben und Sozial ganz unten. Da kam Fach, Methode, Sozial. Ich glaube, das Thema hat sich ein Stück weit rumgedreht, gerade weil die Leute halt nicht mehr alleine für sich im stillen Kämmerlein arbeiten, sondern Teil eines Teams sind.“ (Interviewpartner*in 2, 3. Quartal 2019)

Gleichwohl dokumentiert die Literatur, dass historisch gesehen den Team- und Kommunikationsfähigkeiten eine wachsende Bedeutung zugesprochen wurde (vgl. Kaiser 2006). Die folgende Interviewaussage macht dies deutlich:

„Die mussten sich fast noch öfter austauschen als heute. Denn heute kann ich mir das
Modell vom Kollegen runterladen und mir das an meinem linken Bildschirm anschauen
und dann weiß ich ganz genau, wo meine Übergangspunkte sind. Als ich noch mit der
Hand gezeichnet habe - und das war ja eine unglaubliche Arbeit -, habe ich natürlich
auch lange erst mal nichts, brauchte ich kein Input, weil ich eben auch lange brauchte;
aber wenn ich das Nachbarteil sehen wollte, was vielleicht der Kollege zeichnet, musste
ich ja ganz genau hinschauen. Und da war ich dann auch gezwungen, mich mit dem
zusammen vor sein Brett zu stellen und zu schauen: Was machst du da? Also da
glaube ich mal ehrlich, da mussten die sich früher mindestens genauso, wenn nicht
sogar intensiver austauschen, weil die nämlich sonst am Bedarf vorbei gezeichnet
haben, und das war wie gesagt richtig viel Arbeit. (Interviewpartner*in 3, 4. Quartal
2019)

Allgemein wird die Sozialkompetenz sehr hoch eingeschätzt, auch unter Berücksichtigung interkultureller und internationaler Aspekte. 
Einige der Aussagen bezogen sich auf kein konkretes Kompetenzfeld. Sie spielen jedoch eine Rolle für die Unternehmen in Bezug auf die Digitalisierung und die sich wandelnden Tätigkeiten. Das Systemdenken, also die Fähigkeit, ganzheitlich zu denken und Zusammenhänge zu erkennen, sowie die Fähigkeit, vernetzt und interdisziplinär zu arbeiten, treten immer mehr in den Vordergrund (siehe Abb. 2). Darunter wird sowohl die enge Zusammenarbeit mit Kolleg*innen aus anderen Fachdisziplinen als auch ein Grundverständnis der Elektrik, Elektronik, Regelungstechnik, Softwareentwicklung oder Datenanalyse und in geringerem Maße auch der wirtschaftlichen oder (datenschutz)rechtlichen Fragen verstanden. Die Wertschätzung der Bereitschaft zum lebenslangen Lernen überrascht daher nicht:

„Das muss man sich dann eben, er hat es eben auch schon gesagt, der Wille, sich Neues aneignen zu wollen. Dass man eben sich auch mal schlau macht. Die Welt ist im Wandel, immer wieder was Neues. Gerade die Technik, die geht ja immer weiter nach vorne, und da muss man natürlich am Ball bleiben." (Interviewpartner*in 6, 3. Quartal 2019)

\section{Diskussion: Implikationen für Hochschulcurricula und weiterer Untersuchungsbedarf}

Bei der Analyse der Arbeitgebendenperspektive lässt sich feststellen, dass es eine klare Erwartungshaltung an den Wandel der Berufs- oder Tätigkeitsprofile der Maschinenbauingenieur*innen und damit entsprechend den Kompetenzanforderungen aufgrund der Digitalisierung gibt. Während die Arbeitgebenden ein unterschiedliches Verständnis der Kompetenzen aus dem HQR-Modell aufweisen sowie unterschiedliche Prioritäten setzen, sind sie sich darüber einig, dass grundlegende Fachkompetenzen einer Kerndisziplin nach wie vor ein Muss darstellen und dass die Nachfrage nach fachübergreifender Kommunikation sowie nach fachübergreifenden Fähigkeiten steigt. Während Kommunikationsfähigkeiten als überfachliche oder Sozialkompetenzen gesehen werden, sind fachübergreifende Fähigkeiten aus methodologischer Sicht (Systemdenken) oder aus Sicht anderer Fachdisziplinen zu erwarten. So setzt beispielsweise die Datenkompetenz der Ingenieur*innen Statistik- und Programmierkenntnisse voraus.

Unsere Untersuchung weist darauf hin, dass die Absolvierendenprofile eines durch die Digitalisierung veränderungsbedingten Arbeitsmarktchecks bedürfen. Einschränkend wirken dabei die ressourcenintensive Einbeziehung der Sichtweisen der Arbeitgebenden bei der Curriculumentwicklung und die uneinheitliche 
Operationalisierung der Qualifikations- und Kompetenzanforderungen. Die Verwendung einer einheitlichen Methodik fördert die Vergleichbarkeit von Untersuchungsergebnissen. So lassen sich unsere Ergebnisse mit Heidling et al. (2019) vergleichen, da sie dasselbe Kompetenzmodell verwenden, obwohl sie sich nicht ausdrücklich auf den HQR beziehen. Auch hier wird im ingenieurwissenschaftlichen Anforderungsprofil von fachlich-methodischen Grundlagen in einer Ingenieurdisziplin ausgegangen (zum Beispiel Maschinenbau) und nach weiteren querliegenden fachlichen Grundlagen aus anderen Disziplinen (insbesondere Elektrotechnik und Informatik) sowie nach einer ganzheitlichen Denkweise gefragt. Um vergleichende Erkenntnisse über die Rolle der Digitalisierung in anderen Disziplinen (wie Wirtschafts-, Sozialwissenschaften etcetera) zu gewinnen, ist es erforderlich, weitere Studien auf Grundlage des HQRs durchzuführen.

Dadurch, dass sich die konkrete Curriculumentwicklung im Hoheitsgebiet der Lehrenden befindet, prüfen wir derzeit deren Wahrnehmung der aktuellen und erwarteten Absolvierendenkompetenzen und anschließend konfrontieren wir sie mit den Ansichten der Arbeitgebenden. Erste Analysen zeigen, dass die Lehrenden die Erwartungen der Arbeitgebenden in Bezug auf Industrie 4.0 möglicherweise überschätzen. Der Hochschulabschluss wird von den Arbeitgebenden als Signal für eine grundlegende Humankapitalbildung gesehen, während weitere Spezialisierungen branchen- oder unternehmensbedingt on the Job erfolgen. Des Weiteren herrscht unter den Lehrenden eine gewisse Unsicherheit bezüglich der Schwerpunktsetzung bei den digitalisierungsbezogenen fachübergreifenden Fähigkeiten. Da die Digitalisierung zukünftig die Trennschärfe zwischen den Berufsprofilen der Ingenieurwissenschaftler*innen aufhebt und eine fachübergreifende Austausch- und Lernfähigkeit fordert, ist das ein weiteres Argument dafür, den Dialog mit den Arbeitgebenden bezüglich der Entwicklung zukunftsfähiger Curricula zu suchen. ${ }^{6}$

\section{Literatur}

acatech. (Hrsg.). (2016). Kompetenzentwicklungsstudie Industrie 4.0 - Erste Ergebnisse und Schlussfolgerungen. München: Acatech.

\footnotetext{
${ }^{6}$ Dieser Beitrag ist in leicht geänderter Form im Tagungsband Kompetenzen für die Digitale Transformation 2020 erschienen: Stambolieva, M. (2021). Digitalisierung im Maschinenbau - Beruf im Wandel. In L. Lehmann, D. Engelhardt \& W. Wilke (Hrsg.), Kompetenzen für die Digitale Transformation 2020 (S. 21-36). Berlin: Springer Vieweg.
} 
Acemoglu, D., \& Autor, D. (2011). Skills, tasks and technologies: Implications for employment and earnings. In O. Ashenfelter \& D. E. Card (Hrsg.), Handbook of labor economics, 4 (S. 1043-1171). Amsterdam: Elsevier.

Allmendinger, J. (1989). Career mobility dynamics: A comparative analysis of the United States, Norway and West Germany. Berlin: Max-Planck-Institut für Bildungsforschung.

Allmendinger, J., \& Leibfried, S. (2003). Education and the welfare state: The four worlds of competence production. Journal of European Social Policy, 13(1), 63-81.

Arrow, K. J. (1973). The theory of discrimination. In O. C. Ashenfelter \& A. Rees (Hrsg.), Discrimination in labor markets (S. 3-33). Princeton: Princeton University Press.

Becker, G. S. (1962). Investment in human capital: A theoretical analysis. Journal of Political Economy, 70(5), 9-49.

BMAS. (2017). Weissbuch Arbeiten 4.0. Potsdam: bud Potsdam.

Bourdieu, P. (1983). Ökonomisches Kapital, kulturelles Kapital, soziales Kapital. In R. Kreckel (Hrsg.), Soziale Ungleichheiten. Sonderheft 2 der Sozialen Welt (S. 183-198). Göttingen: Schwartz.

Bundesagentur für Arbeit. (2020). Ingenieur/-in Maschinenbau. Tätigkeit nach Studium. Trends. https://berufenet.arbeitsagentur.de/berufenet/faces/index?path=null/kurzbeschrei bung/trends\&dkz=58731. Zugegriffen: 7. Apr. 2020.

Dengler, K., \& Matthes, B. (2018). Substituierbarkeitspotenziale von Berufen: Wenige Berufe halten mit der Digitalisierung Schritt. IAB Kurzbericht, 4. http://doku.iab.de/kurzber/ 2018/kb0418.pdf. Zugegriffen: 7. Apr. 2020.

Dilger, B., Ebert, A., \& Landmann, M. (2012). The Missing Link?! Verbindungen zwischen konzeptionellen und empirischen Zugängen zur Kompetenzmodellierung an Hochschulen. Zeitschrift für Hochschulentwicklung, 7(4), 71-84.

EHEA. (2015). Ministerial Conference Yerevan 2015. https://www.ehea.info/cid101764/min isterial-conference-yerevan-2015.html. Zugegriffen: 3. Apr. 2020.

EHEA. (2016). Employability of graduates. EHEA introductory material. https://www.ehea. info/pid34423-cid102525/employability-of-graduates.html. Zugegriffen: 3. Apr. 2020.

Eimer, A., Knauer, J., Kremer, I., Nowak, T., \& Schröder, A. (2019). Employability als ein Ziel des Universitätsstudiums. Grundlagen, Methoden, Wirkungsanalyse. Bielefeld: wbv Media.

Erpenbeck, J., von Rosenstiel, L., Grote. S., \& Sauter, W. (Hrsg.) (2017). Handbuch Kompetenzmessung. Erkennen, verstehen und bewerten von Kompetenzen in der betrieblichen, pädagogischen und psychologischen Praxis (3., überarbeitete und erweiterte Aufl.). Stuttgart: Schäffer-Peschel.

European Commission/EACEA/Eurydice. (2018). The European higher education area in 2018: Bologna process implementation report. Luxemburg: Publications Office of the European Union.

Fugate, M., Kinicki, A. J., \& Ashforth, B. E. (2004). Employability: A psycho-social construct, its dimensions, and applications. Journal of Vocational Behavior, 65, 14-38.

Gerholz, K., \& Sloane, P. F. E. (2011). Lernfelder als universitäres Curriculum? Eine hochschuldidaktische Adaption. Bwp@Berufs- und Wirtschaftspädagogik online, 20, $1-23$.

Gillies, D. (2011). State-education as high-yield investment: human capital theory in European policy discourse. Journal of Pedagogy, 2(2), 224-245. https://doi.org/10.2478/v10159011-0011-3. 
Goos, M., Manning, A., \& Salomons, A. (2014). Explaining job polarization: Routine-biased technological change and offshoring. The American Economic Review, 104(8), 25092526.

Gössling, B., \& Luft, B. E. (2019). Handlungsorientierung von Hochschullehrenden im Umgang mit der Entwicklung lernergebnisbasierter Curricula. Zeitschrift für Hochschulentwicklung, 14(1), 57-78.

Gottburgsen, A., Wannemacher, K., Wernz, J., \& Willige, J. (2019). Ingenieurausbildung für die Digitale Transformation. Zukunft durch Veränderung. Düsseldorf: VDI Verein Deutscher Ingenieure e. V.

Heidling E., Meil, P., Neumer, J., Porschen-Hueck, S., Schmierl, K., Sopp, P., \& Wagner A. (2019). Ingenieurinnen und Ingenieure für Industrie 4.0. München: Impuls-Stiftung des VDMA.

Hessler, G. (2013). Employability in der Hochschule? Analysen zur Perspektive von Studierenden der Sozial- und Geisteswissenschaften. Zeitschrift für Hochschulentwicklung, $8(1), 45-59$.

HRK. (2020a). Beschäftigungsbefähigung. Glossar der Studienreform. https://www.hrknexus.de/glossar-der-studienreform/begriff/beschaeftigungsbefaehigung/. Zugegriffen: 3 . Apr. 2020.

HRK. (2020b). Kompetenzorientierung. Glossar der Studienreform. https://www.hrk-nexus. de/glossar-der-studienreform/begriff/kompetenzorientierung/. Zugegriffen: 3. Apr. 2020.

Kaiser, W. (2006). Ingenieure in der Bundesrepublik Deutschland. In W. Kaiser \& W. König (Hrsg.), Geschichte des Ingenieurs. Ein Beruf in sechs Jahrtausenden (S. 233-267). München: Hanser.

KMK. (2017a). Qualifikationsrahmen für deutsche Hochschulabschlüsse. Beschluss der Kultusministerkonferenz vom 16.02.2017. https:/www.kmk.org/fileadmin/Dateien/veroef fentlichungen_beschluesse/2017/2017_02_16-Qualifikationsrahmen.pdf. Zugegriffen: 3 . Apr. 2020.

KMK. (2017b). Musterrechtsverordnung gemäß Artikel 4 Absätze 1-4 Studienakkreditierungsstaatsvertrag. Beschluss der Kultusministerkonferenz vom 07.12.2017. https:// www.akkreditierungsrat.de/de/media/23. Zugegriffen: 3. Apr. 2020.

Kurer, T., \& Palier, B. (2019). Shrinking and shouting: The political revolt of the declining middle in times of employment polarization. Research \& Politics, 6, 1. https://doi.org/10. $1177 / 2053168019831164$.

Lautenbach, C., Toepper, M., Zlatkin-Troitschanskaia, O., \& Pant, H. A. (2017). Akademische Kompetenzen von Studierenden - Modellierungs- und Erfassungsansätze. In U. Schmidt (Hrsg.), Handbuch Qualität in Studium und Lehre (S. 1-30). Berlin: DUZ.

Nedelkoska, L., \& Quintini, G. (2018). Automation, skills use and training. OECD social, employment and migration Working Papers, 202. https://doi.org/10.1787/1815199X.

OECD. (2019). OECD employment outlook 2019: The future of work. Paris: OECD Publishing.

Pfeiffer, S., \& Suphan, A. (2018). Industrie 4.0 und Erfahrung - das unterschätzte Innovationsund Gestaltungspotenzial der Beschäftigten im Maschinen- und Automobilbau. In H. Hirsch-Kreinsen, P. Ittermann, \& J. Niehaus (Hrsg.), Digitalisierung industrieller Arbeit. Die Vision Industrie 4.0 und ihre sozialen Herausforderungen, (2. Aufl., S. 275-301). Baden-Baden: edition sigma in der Nomos Verlagsgesellschaft. 
Schubarth, W., Speck, K., Ulbricht, J., Dudziak, I., \& Zylla, B. (2014). Employability und Praxisbezüge im wissenschaftlichen Studium. HRK Fachgutachten. https://www.hrknexus.de/fileadmin/redaktion/hrk-nexus/07-Downloads/07-02-Publikationen/Fachgutac hten_Employability-Praxisbezuege.pdf. Zugegriffen: 3. Apr. 2020.

Schüller, K. \& Busch, P. (2019). Data literacy: Ein systematic review (Arbeitspapier, 46). Berlin: Hochschulforum Digitalisierung. https://doi.org/10.5281/zenodo.3484583.

Schüller, K., Busch, P., \& Hindinger, C. (2019). Future Skills: Ein Framework für Data Literacy (Arbeitspapier, 47). Berlin: Hochschulforum Digitalisierung. https://doi.org/10. 5281/zenodo.3349865.

Spence, M. (1973). Job market signalling. Quarterly Journal of Economics, 87(3), 355-374.

Statista. (2020). Anzahl der Beschäftigten im deutschen Maschinenbau in den Jahren 1991 bis 2018. https://de.statista.com/statistik/daten/studie/30821/umfrage/beschaeftigteim-maschinenbau-in-deutschland-seit-1991/. Zugegriffen: 7. Apr. 2020.

Stifterverband. (2016). Hochschul-Bildungs-Report 2020: Hochschulbildung für die Arbeitswelt 4.0. Essen: Stifterverband für die deutsche Wissenschaft e. V.

Teichler, U. (2018). Higher education and graduate employment: Changing conditions and challenges. INCHER Working Paper, 10. https://doi.org/10.17906/INCHER.0002.

WEF (2016). The 10 skills you need to thrive in the fourth industrial revolution. https://www.weforum.org/agenda/2016/01/the-10-skills-you-need-to-thrive-in-thefourth-industrial-revolution/. Zugegriffen: 4. Febr. 2020.

Open Access Dieses Kapitel wird unter der Creative Commons Namensnennung 4.0 International Lizenz (http://creativecommons.org/licenses/by/4.0/deed.de) veröffentlicht, welche die Nutzung, Vervielfältigung, Bearbeitung, Verbreitung und Wiedergabe in jeglichem Medium und Format erlaubt, sofern Sie den/die ursprünglichen Autor(en) und die Quelle ordnungsgemäß nennen, einen Link zur Creative Commons Lizenz beifügen und angeben, ob Änderungen vorgenommen wurden.

Die in diesem Kapitel enthaltenen Bilder und sonstiges Drittmaterial unterliegen ebenfalls der genannten Creative Commons Lizenz, sofern sich aus der Abbildungslegende nichts anderes ergibt. Sofern das betreffende Material nicht unter der genannten Creative Commons Lizenz steht und die betreffende Handlung nicht nach gesetzlichen Vorschriften erlaubt ist, ist für die oben aufgeführten Weiterverwendungen des Materials die Einwilligung des jeweiligen Rechteinhabers einzuholen.

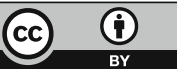

